Supporting Information for

\title{
5,5- and 5,6-Membered Spirocyclic Indolinone Hit-Finding Libraries
}

Peter Meier*, Nicole Battaglia, Peter Ertl, Bernard Pirard

Global Discovery Chemistry, Novartis Institutes for Biomedical Research, CH-4056 Basel, Switzerland

*Corresponding author email: peter.meier@novartis.com

\section{Contents}

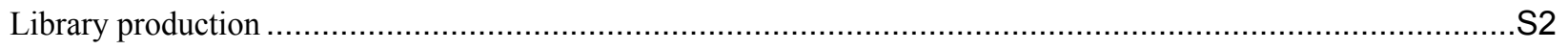

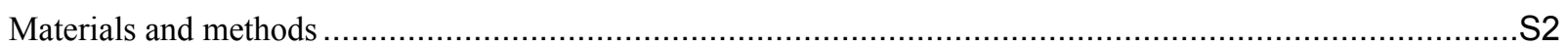

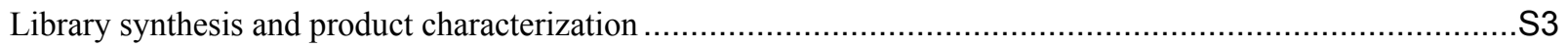

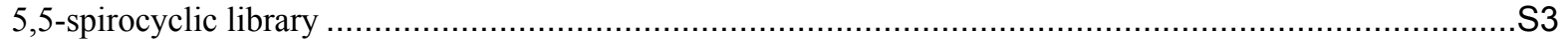

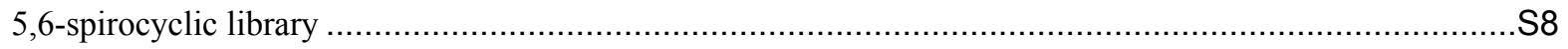

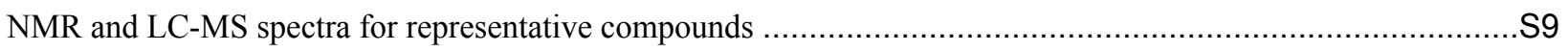

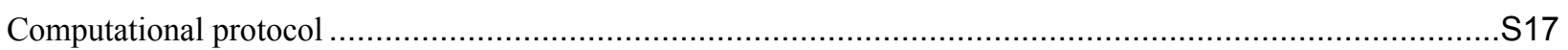




\section{Library production}

\section{Materials and methods}

Shaker: Heidolph Titramax 100 with 96 position Remp racks. Microwave factory. ${ }^{12}$

Purification:

5,5-spirocyclic library: reverse phase HPLC done with Waters 600 HPLC pump with DAD detector Waters 2487 UV and Guilson Autosampler; eluent: 0.1\% TFA in water / 0.1\% TFA in acetonitrile, 95/5 for 1 min, then to 5/95 in 6 min; flow: $15 \mathrm{~mL} / \mathrm{min}$; column: SunFire C18 OBD Prep Column, $100 \AA,, 5 \mu \mathrm{m}, 30 \mathrm{~mm} \times 100 \mathrm{~mm}$; 1 fraction (max. 10 $\mathrm{mL}$ ) was collected per injection.

5,6-spirocyclic library: reverse phase HPLC done with Waters 2525 HPLC pump with DAD detector Waters 2487

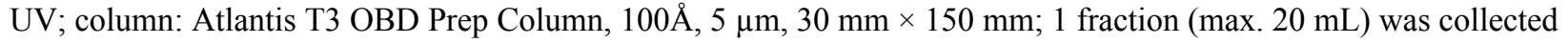
per injection. Eluents/gradients: according to the pre-analysis of the reaction mixture, the optimal gradient was selected from the following choice:

Gradient 1: $0.1 \%$ TFA in water / $0.1 \%$ TFA in acetonitrile, 100/0 for $0.5 \mathrm{~min}$, from 100/0 to $90 / 10$ in $4.5 \mathrm{~min}$, then to $0 / 100$ in $0.1 \mathrm{~min}$; flow: $60 \mathrm{~mL} / \mathrm{min}$.

Gradient 2: $0.1 \%$ TFA in water / $0.1 \%$ TFA in acetonitrile, $100 / 0$ to $90 / 10$ in $0.5 \mathrm{~min}$, to $40 / 60$ in $4.5 \mathrm{~min}$, then to 0/100 in $0.1 \mathrm{~min}$; flow: $60 \mathrm{~mL} / \mathrm{min}$.

Gradient 3: $0.1 \%$ TFA in water / $0.1 \%$ TFA in acetonitrile, $100 / 0$ to $80 / 20$ in $0.5 \mathrm{~min}$, to $30 / 70$ in $4.5 \mathrm{~min}$, then to 0/100 in $0.1 \mathrm{~min}$; flow: $60 \mathrm{~mL} / \mathrm{min}$.

Gradient $4: 0.1 \%$ TFA in water $/ 0.1 \%$ TFA in acetonitrile, $100 / 0$ to $70 / 30$ in $0.5 \mathrm{~min}$, to $20 / 80$ in $4.5 \mathrm{~min}$, then to 0/100 in $0.1 \mathrm{~min}$; flow: $60 \mathrm{~mL} / \mathrm{min}$.

Gradient 5: $0.1 \%$ TFA in water / $0.1 \%$ TFA in acetonitrile, $100 / 0$ to $60 / 40$ for $0.5 \mathrm{~min}$, to $5 / 95$ in $4.5 \mathrm{~min}$, then to 0/100 in $0.1 \mathrm{~min}$; flow: $60 \mathrm{~mL} / \mathrm{min}$.

Analytical HPLC conditions used for the quality control of the compounds:

Waters Acquity UPLC system; eluent: 0.1\% TFA in water / 0.1\% TFA in acetonitrile, 95/5 for 0.2 min, then to 5/95 in $0.9 \mathrm{~min}, 0.3 \mathrm{~min}$ at 5/95; UV observation wavelength: $214 \mathrm{~nm}$; column: for 5,5-spirocyclic library: Acquity UPLC HSS T3 $1.8 \mu \mathrm{m}$; for 5,6-spirocyclic library: Acquity Cortecs C18 $1.6 \mu \mathrm{m}$.

${ }^{1} \mathrm{H}$ and ${ }^{13} \mathrm{C}$ spectra were obtained on Bruker $400 \mathrm{MHz}$ instrument. ${ }^{1} \mathrm{H}$ and ${ }^{13} \mathrm{C}$ chemical shifts are reported in delta $(\delta)$ units in parts per million (ppm) relative to the TMS and relative solvent $\mathrm{CDCl}_{3}$ and $\mathrm{MeOD}$. Coupling constants are recorded in Hertz $(\mathrm{Hz})$. 


\section{Library synthesis and product characterization}

\section{5,5-spirocyclic library}

Library synthesis<smiles>[R7]C1NC(C(=O)OC)CC12C(=O)Nc1ccccc12</smiles>

2<smiles>[R20]O[R10]([H])([H])O[R20]</smiles>

13

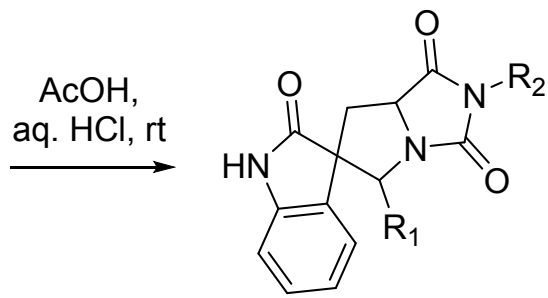

3

The methyl-2'-alkyl-2-oxospiro[indoline-3,3'-pyrrolidine]-5'-carboxylates 2 were prepared according to literature. ${ }^{5}$ The derivatization of these intermediates were performed in glass tubes within 96-position Remp racks on the shaker: A $3 \mathrm{~mL}$ glass tube was charged with a mixture of the intermediate $2(0.05 \mathrm{mmol})$ and isocyanate $12(0.055 \mathrm{mmol})$ in dichloromethane $(1.5 \mu \mathrm{L}$; prepared as stock solutions). The reaction mixture was shaken overnight at room temperature $\left(20-25^{\circ} \mathrm{C}\right)$. Then acetic acid $(100 \mu \mathrm{L})$ was added and the dichloromethane part of the reaction was carefully blown away (maximum one hour and no turbulence of the mixture).

The crude was dissolved in acetic acid $(300 \mu \mathrm{L})$. Then $3 \mathrm{~N} \mathrm{HCl}(300 \mu \mathrm{L})$ was added and the reaction mixture was shaken overnight at room temperature $\left(20-25{ }^{\circ} \mathrm{C}\right)$.

The reaction mixture was directly purified by preparative LC/MS ( $s$. $t_{R}$ in the following tables). All the obtained products 3 were characterized by HPLC (UV) and MS.

Product characterization

$\begin{array}{lccccc}\text { Product 3 } & \begin{array}{c}\text { Collected } \\ {[\mathrm{MW}+\mathrm{H}]^{+}}\end{array} & \mathrm{t}_{\mathrm{R}}(\mathrm{min}) & \text { Yield }(\mathrm{mg}) & \text { Yield (\%) } & \text { Purity (\%, UV) } \\ \mathbf{3}\{1,1,1\} & 390.46 & 5.97 & 5.4 & 28 & 100 \\ \mathbf{3}\{1,1,2\} & 404.49 & 6.28 & 5.5 & 27 & 100 \\ \mathbf{3}\{1,1,3\} & 404.49 & 6.26 & 5.5 & 27 & 100 \\ \mathbf{3}\{1,1,4\} & 404.49 & 6.26 & 5.3 & 26 & 100 \\ \mathbf{3}\{1,1,5\} & 420.48 & 6.04 & 4.5 & 21 & 100 \\ \mathbf{3}\{1,1,6\} & 420.48 & 6.04 & 5.6 & 27 & 100 \\ \mathbf{3}\{1,1,7\} & 408.45 & 6.06 & 4.6 & 23 & 100 \\ \mathbf{3}\{1,1,9\} & 408.45 & 5.91 & 5.4 & 26 & 100 \\ \mathbf{3}\{1,1,11\} & 424.90 & 6.15 & 4.5 & 21 & 100 \\ \mathbf{3}\{1,1,12\} & 480.54 & 5.40 & 6.4 & 27 & 100 \\ \mathbf{3}\{1,1,14\} & 404.49 & 6.43 & 4.8 & 24 & 100 \\ \mathbf{3}\{1,1,15\} & 448.50 & 6.00 & 6.4 & 29 & 100 \\ \mathbf{3}\{1,1,16\} & 410.51 & 6.11 & 5.4 & 26 & 100 \\ \mathbf{3}\{1,1,17\} & 328.39 & 5.10 & 3.5 & 21 & 100 \\ \mathbf{3}\{1,1,18\} & 342.41 & 5.79 & 3.4 & 20 & 100 \\ \mathbf{3}\{1,1,20\} & 370.47 & 6.46 & 4.3 & 23 & 100 \\ \mathbf{3}\{1,1,21\} & 382.48 & 6.65 & 3.6 & 19 & 100 \\ \mathbf{3}\{1,1,22\} & 340.40 & 5.37 & 4.5 & 26 & 100\end{array}$




\begin{tabular}{|c|c|c|c|c|c|}
\hline Product 3 & $\begin{array}{c}\text { Collected } \\
{[\mathrm{MW}+\mathrm{H}]^{+}}\end{array}$ & $\mathrm{t}_{\mathrm{R}}(\min )$ & Yield (mg) & Yield (\%) & Purity $(\%, U V)$ \\
\hline $\mathbf{3}\{1,5,1\}$ & 404.49 & 6.30 & 4.4 & 22 & 100 \\
\hline $\mathbf{3}\{1,5,2\}$ & 418.51 & 6.57 & 3.8 & 18 & 100 \\
\hline $\mathbf{3}\{1,5,3\}$ & 418.51 & 6.61 & 4.3 & 21 & 100 \\
\hline $\mathbf{3}\{1,5,4\}$ & 418.51 & 6.57 & 4.4 & 21 & 100 \\
\hline $3\{1,5,6\}$ & 434.51 & 6.32 & 4.1 & 19 & 100 \\
\hline $\mathbf{3}\{1,5,7\}$ & 422.48 & 6.35 & 4.3 & 20 & 100 \\
\hline $3\{1,5,9\}$ & 422.48 & 6.30 & 4.0 & 19 & 100 \\
\hline $3\{1,5,14\}$ & 418.51 & 6.69 & 3.8 & 18 & 100 \\
\hline $\mathbf{3}\{1,5,15\}$ & 462.52 & 6.28 & 4.8 & 21 & 100 \\
\hline $3\{1,5,16\}$ & 424.54 & 6.37 & 3.7 & 17 & 100 \\
\hline $3\{1,5,17\}$ & 342.41 & 5.47 & 3.8 & 22 & 100 \\
\hline $3\{1,5,19\}$ & 370.47 & 6.30 & 3.0 & 16 & 100 \\
\hline $3\{1,5,22\}$ & 354.42 & 5.75 & 3.4 & 19 & 100 \\
\hline $3\{1,7,1\}$ & 430.52 & 6.57 & 6.8 & 32 & 100 \\
\hline $\mathbf{3}\{1,7,2\}$ & 444.55 & 6.85 & 5.0 & 22 & 100 \\
\hline $\mathbf{3}\{1,7,3\}$ & 444.55 & 6.92 & 5.1 & 23 & 100 \\
\hline $\mathbf{3}\{1,7,4\}$ & 444.55 & 6.86 & 5.4 & 24 & 100 \\
\hline $\mathbf{3}\{1,7,6\}$ & 460.55 & 6.58 & 6.2 & 23 & 85 \\
\hline $3\{1,7,14\}$ & 444.55 & 6.97 & 5.8 & 26 & 100 \\
\hline $\mathbf{3}\{1,7,15\}$ & 488.56 & 6.55 & 6.9 & 28 & 100 \\
\hline $3\{1,7,16\}$ & 450.58 & 6.72 & 5.8 & 26 & 100 \\
\hline $\mathbf{3}\{1,7,17\}$ & 368.45 & 5.89 & 4.5 & 24 & 100 \\
\hline $3\{1,7,18\}$ & 382.48 & 6.47 & 5.1 & 27 & 100 \\
\hline $3\{1,7,19\}$ & 396.51 & 6.72 & 5.7 & 29 & 100 \\
\hline $3\{1,7,20\}$ & 410.53 & 7.03 & 4.3 & 21 & 100 \\
\hline $3\{1,7,22\}$ & 380.46 & 6.13 & 4.7 & 25 & 100 \\
\hline $3\{1,8,1\}$ & 466.56 & 6.74 & 7.4 & 32 & 100 \\
\hline $\mathbf{3}\{1,8,6\}$ & 496.58 & 6.84 & 6.0 & 24 & 100 \\
\hline $3\{1,8,8\}$ & 484.55 & 6.75 & 6.9 & 28 & 100 \\
\hline $\mathbf{3}\{1,8,9\}$ & 484.55 & 6.66 & 7.5 & 31 & 100 \\
\hline $3\{1,8,14\}$ & 480.58 & 7.06 & 3.8 & 16 & 100 \\
\hline $3\{1,8,16\}$ & 486.61 & $6.75 / 6.92$ & 7.0 & 29 & 100 \\
\hline $3\{1,8,17\}$ & 404.49 & 6.02 & 5.4 & 27 & 100 \\
\hline $3\{1,8,18\}$ & 418.51 & 6.60 & 4.4 & 21 & 100 \\
\hline $3\{1,8,19\}$ & 432.54 & 6.72 & 6.1 & 28 & 100 \\
\hline $3\{1,8,20\}$ & 446.57 & 7.12 & 6.5 & 29 & 100 \\
\hline $3\{1,8,22\}$ & 416.50 & 6.28 & 4.8 & 23 & 100 \\
\hline $\mathbf{3}\{2,1,2\}$ & 404.49 & 6.28 & 7.4 & 37 & 100 \\
\hline $\mathbf{3}\{2,1,3\}$ & 404.49 & 6.26 & 6.7 & 33 & 100 \\
\hline $3\{2,1,4\}$ & 404.49 & 6.26 & 6.9 & 34 & 100 \\
\hline $\mathbf{3}\{2,1,7\}$ & 408.45 & 6.06 & 8.1 & 40 & 100 \\
\hline
\end{tabular}




\begin{tabular}{|c|c|c|c|c|c|}
\hline Product 3 & $\begin{array}{c}\text { Collected } \\
{[\mathrm{MW}+\mathrm{H}]^{+}}\end{array}$ & $\mathrm{t}_{\mathrm{R}}(\min )$ & Yield (mg) & Yield (\%) & Purity $(\%, U V)$ \\
\hline $\mathbf{3}\{2,1,8\}$ & 408.45 & 6.08 & 6.8 & 30 & 89 \\
\hline $3\{2,1,9\}$ & 408.45 & 5.93 & 8.9 & 37 & 85 \\
\hline $3\{2,1,11\}$ & 424.90 & $6.24 / 6.39$ & 6.8 & 32 & 100 \\
\hline $3\{2,1,12\}$ & 480.54 & 5.47 & 8.6 & 36 & 100 \\
\hline $\mathbf{3}\{2,1,13\}$ & 380.42 & 5.58 & 6.1 & 29 & 90 \\
\hline $3\{2,1,14\}$ & 404.49 & 6.43 & 7.3 & 36 & 100 \\
\hline $\mathbf{3}\{2,1,15\}$ & 448.50 & 5.98 & 8.2 & 37 & 100 \\
\hline $\mathbf{3}\{2,1,16\}$ & 410.51 & 6.13 & 7.9 & 38 & 100 \\
\hline $3\{2,1,17\}$ & 328.39 & 5.12 & 6.6 & 40 & 100 \\
\hline $3\{2,1,18\}$ & 342.41 & 5.79 & 6.4 & 37 & 100 \\
\hline $3\{2,1,19\}$ & 356.44 & 6.04 & 5.8 & 33 & 100 \\
\hline $3\{2,1,20\}$ & 370.47 & 6.44 & 7.1 & 38 & 100 \\
\hline $\mathbf{3}\{2,1,21\}$ & 382.48 & 6.67 & 6.0 & 31 & 100 \\
\hline $3\{2,1,22\}$ & 340.40 & 5.40 & 7.6 & 45 & 100 \\
\hline $3\{2,2,4\}$ & 376.43 & 5.86 & 8.6 & 41 & 90 \\
\hline $\mathbf{3}\{2,2,8\}$ & 380.39 & 5.62 & 3.1 & 14 & 85 \\
\hline $3\{2,2,13\}$ & 352.37 & 5.19 & 5.9 & 28 & 85 \\
\hline $3\{2,2,16\}$ & 382.46 & 5.51 & 4.2 & 22 & 100 \\
\hline $\mathbf{3}\{2,2,17\}$ & 300.33 & 4.43 & 6.8 & 45 & 100 \\
\hline $3\{2,2,18\}$ & 314.36 & 5.03 & 4.5 & 26 & 92 \\
\hline $3\{2,2,19\}$ & 328.39 & 5.47 & 6.9 & 42 & 100 \\
\hline $3\{2,2,20\}$ & 342.41 & 5.91 & 6.3 & 37 & 100 \\
\hline $3\{2,2,22\}$ & 312.34 & 4.77 & 8.3 & 53 & 100 \\
\hline $\mathbf{3}\{2,3,1\}$ & 376.43 & 5.75 & 7.8 & 41 & 100 \\
\hline $\mathbf{3}\{2,3,2\}$ & 390.46 & 6.11 & 7.6 & 39 & 100 \\
\hline $\mathbf{3}\{2,3,3\}$ & 390.46 & 6.00 & 6.1 & 31 & 100 \\
\hline $\mathbf{3}\{2,3,4\}$ & 390.46 & 6.00 & 7.2 & 37 & 100 \\
\hline $3\{2,3,6\}$ & 406.46 & 5.84 & 8.5 & 36 & 87 \\
\hline $\mathbf{3}\{2,3,8\}$ & 394.42 & 6.78 & 8.7 & 44 & 100 \\
\hline $\mathbf{3}\{2,3,9\}$ & 394.42 & 5.67 & 8.2 & 42 & 100 \\
\hline $3\{2,3,11\}$ & 410.88 & $6.02 / 6.19$ & 5.0 & 24 & 100 \\
\hline $3\{2,3,12\}$ & 466.51 & 5.18 & 7.0 & 30 & 100 \\
\hline $\mathbf{3}\{2,3,13\}$ & 366.39 & 5.30 & 7.1 & 39 & 100 \\
\hline $3\{2,3,14\}$ & 390.46 & 6.21 & 8.2 & 42 & 100 \\
\hline $3\{2,3,15\}$ & 434.47 & 5.75 & 9.3 & 43 & 100 \\
\hline $\mathbf{3}\{2,3,16\}$ & 396.48 & 5.86 & 6.1 & 31 & 100 \\
\hline $3\{2,3,17\}$ & 314.36 & 4.76 & 7.3 & 46 & 100 \\
\hline $\mathbf{3}\{2,3,18\}$ & 328.39 & 5.47 & 3.5 & 21 & 100 \\
\hline $3\{2,3,19\}$ & 342.41 & 5.73 & 5.5 & 32 & 100 \\
\hline $\mathbf{3}\{2,3,20\}$ & 356.44 & 6.13 & 5.8 & 33 & 100 \\
\hline
\end{tabular}




\begin{tabular}{|c|c|c|c|c|c|}
\hline Product 3 & $\begin{array}{l}\text { Collected } \\
{[\mathrm{MW}+\mathrm{H}]^{+}}\end{array}$ & $\mathrm{t}_{\mathrm{R}}(\min )$ & Yield (mg) & Yield (\%) & Purity $(\%, U V)$ \\
\hline $3\{2,3,21\}$ & 368.45 & 6.40 & 5.2 & 25 & 90 \\
\hline $3\{2,3,22\}$ & 326.37 & 5.09 & 6.7 & 41 & 100 \\
\hline $\mathbf{3}\{2,4,1\}$ & 390.46 & 6.00 & 7.2 & 37 & 100 \\
\hline $\mathbf{3}\{2,4,2\}$ & 404.49 & 6.30 & 6.1 & 30 & 100 \\
\hline $\mathbf{3}\{2,4,3\}$ & 404.49 & 6.31 & 5.6 & 28 & 100 \\
\hline $\mathbf{3}\{2,4,4\}$ & 404.49 & 6.30 & 5.8 & 29 & 100 \\
\hline $\mathbf{3}\{2,4,5\}$ & 420.48 & 6.10 & 5.9 & 28 & 100 \\
\hline $\mathbf{3}\{2,4,6\}$ & 420.48 & 6.10 & 6.5 & 31 & 100 \\
\hline $\mathbf{3}\{2,4,7\}$ & 408.45 & 6.09 & 6.3 & 31 & 100 \\
\hline $\mathbf{3}\{2,4,8\}$ & 408.45 & 6.11 & 6.8 & 33 & 100 \\
\hline $\mathbf{3}\{2,4,9\}$ & 408.45 & 5.93 & 6.4 & 27 & 85 \\
\hline $3\{2,4,11\}$ & 424.90 & 6.31 & 5.8 & 27 & 100 \\
\hline $3\{2,4,12\}$ & 480.54 & 5.53 & 6.9 & 29 & 100 \\
\hline $3\{2,4,13\}$ & 380.42 & 5.60 & 5.2 & 27 & 100 \\
\hline $3\{2,4,14\}$ & 404.49 & 6.47 & 6.4 & 32 & 100 \\
\hline $\mathbf{3}\{2,4,15\}$ & 448.50 & 6.08 & 6.9 & 31 & 100 \\
\hline $3\{2,4,16\}$ & 410.51 & 6.15 & 5.8 & 28 & 100 \\
\hline $3\{2,4,17\}$ & 328.39 & 5.14 & 5.0 & 30 & 100 \\
\hline $\mathbf{3}\{2,4,18\}$ & 342.41 & 5.79 & 4.4 & 26 & 100 \\
\hline $3\{2,4,19\}$ & 356.44 & 6.02 & 5.4 & 30 & 100 \\
\hline $\mathbf{3}\{2,4,20\}$ & 370.47 & 6.44 & 5.7 & 31 & 100 \\
\hline $3\{2,4,22\}$ & 340.40 & 5.49 & 5.2 & 31 & 100 \\
\hline $\mathbf{3}\{2,5,1\}$ & 404.49 & 6.26 & 5.2 & 22 & 85 \\
\hline $\mathbf{3}\{2,5,2\}$ & 418.51 & 6.53 & 6.0 & 29 & 100 \\
\hline $\mathbf{3}\{2,5,3\}$ & 418.51 & 6.53 & 6.2 & 30 & 100 \\
\hline $\mathbf{3}\{2,5,4\}$ & 418.51 & 6.61 & 6.0 & 29 & 100 \\
\hline $\mathbf{3}\{2,5,6\}$ & 434.51 & 6.30 & 5.4 & 25 & 100 \\
\hline $\mathbf{3}\{2,5,7\}$ & 422.48 & 6.33 & 4.8 & 19 & 85 \\
\hline $\mathbf{3}\{2,5,8\}$ & 422.48 & 6.39 & 6.0 & 28 & 100 \\
\hline $\mathbf{3}\{2,5,11\}$ & 438.93 & 6.63 & 4.1 & 19 & 100 \\
\hline $3\{2,5,12\}$ & 494.56 & $5.69 / 6.00$ & 4.2 & 17 & 100 \\
\hline $\mathbf{3}\{2,5,14\}$ & 418.51 & 6.69 & 6.3 & 30 & 100 \\
\hline $3\{2,5,15\}$ & 462.52 & 6.22 & 7.1 & 31 & 100 \\
\hline $3\{2,5,16\}$ & 424.54 & 6.35 & 6.0 & 28 & 100 \\
\hline $3\{2,5,17\}$ & 342.41 & 5.47 & 4.8 & 28 & 100 \\
\hline $\mathbf{3}\{2,5,18\}$ & 356.44 & 6.08 & 3.5 & 20 & 100 \\
\hline $3\{2,5,19\}$ & 370.47 & 6.39 & 4.4 & 24 & 100 \\
\hline $\mathbf{3}\{2,5,20\}$ & 384.49 & 6.66 & 4.0 & 21 & 100 \\
\hline $\mathbf{3}\{2,5,22\}$ & 354.42 & 5.62 & 5.5 & 31 & 100 \\
\hline $3\{2,6,2\}$ & 418.51 & 6.55 & 5.7 & 27 & 100 \\
\hline
\end{tabular}




$\begin{array}{cccccc}\text { Product } 3 & \begin{array}{c}\text { Collected } \\ {[\mathrm{MW}+\mathrm{H}]^{+}}\end{array} & \mathrm{t}_{\mathrm{R}}(\mathrm{min}) & \text { Yield (mg) } & \text { Yield (\%) } & \text { Purity (\%, UV) } \\ & & & & & \\ \mathbf{3}\{2,6,4\} & 418.51 & 6.57 & 5.4 & 26 & 100 \\ \mathbf{3}\{2,6,5\} & 434.51 & 6.36 & 5.9 & 23 & 85 \\ \mathbf{3}\{2,6,6\} & 434.51 & 6.36 & 6.4 & 25 & 85 \\ \mathbf{3}\{2,6,7\} & 422.48 & 6.35 & 5.9 & 24 & 85 \\ \mathbf{3}\{2,6,9\} & 422.48 & 6.19 & 6.4 & 26 & 85 \\ \mathbf{3}\{2,6,11\} & 438.93 & 6.44 & 5.8 & 26 & 100 \\ \mathbf{3}\{2,6,12\} & 494.56 & 5.73 & 7.5 & 30 & 100 \\ \mathbf{3}\{2,6,13\} & 394.45 & 5.86 & 6.4 & 32 & 100 \\ \mathbf{3}\{2,6,14\} & 418.51 & 6.71 & 5.0 & 24 & 100 \\ \mathbf{3}\{2,6,16\} & 424.54 & 6.41 & 9.2 & 43 & 100 \\ \mathbf{3}\{2,6,17\} & 342.41 & 5.49 & 6.2 & 36 & 100 \\ \mathbf{3}\{2,6,18\} & 356.44 & 6.08 & 5.8 & 32 & 100 \\ \mathbf{3}\{2,6,19\} & 370.47 & 6.35 & 5.7 & 31 & 100 \\ \mathbf{3}\{2,6,20\} & 384.49 & 6.68 & 6.0 & 31 & 100 \\ \mathbf{3}\{2,6,21\} & 396.51 & 6.95 & 4.8 & 22 & 90 \\ \mathbf{3}\{2,6,22\} & 354.42 & 5.78 & 6.3 & 35 & 100 \\ \mathbf{3}\{2,7,1\} & 430.52 & 6.53 & 4.4 & 17 & 85 \\ \mathbf{3}\{2,7,2\} & 444.55 & 6.85 & 4.5 & 20 & 100 \\ \mathbf{3}\{2,7,5\} & 460.55 & 6.60 & 5.0 & 22 & 100 \\ \mathbf{3}\{2,7,14\} & 444.55 & 7.01 & 4.6 & 19 & 94 \\ \mathbf{3}\{2,7,16\} & 450.58 & 6.66 & 4.8 & 21 & 100 \\ \mathbf{3}\{2,7,17\} & 368.45 & 5.89 & 3.5 & 19 & 100 \\ \mathbf{3}\{2,7,19\} & 396.51 & 6.68 & 4.5 & 23 & 100 \\ \mathbf{3}\{2,7,20\} & 410.53 & 7.05 & 4.2 & 20 & 100 \\ \mathbf{3}\{2,7,22\} & 380.46 & 6.08 & 4.4 & 23 & 100 \\ & & & & & \end{array}$




\section{5,6-spirocyclic library}

Library synthesis

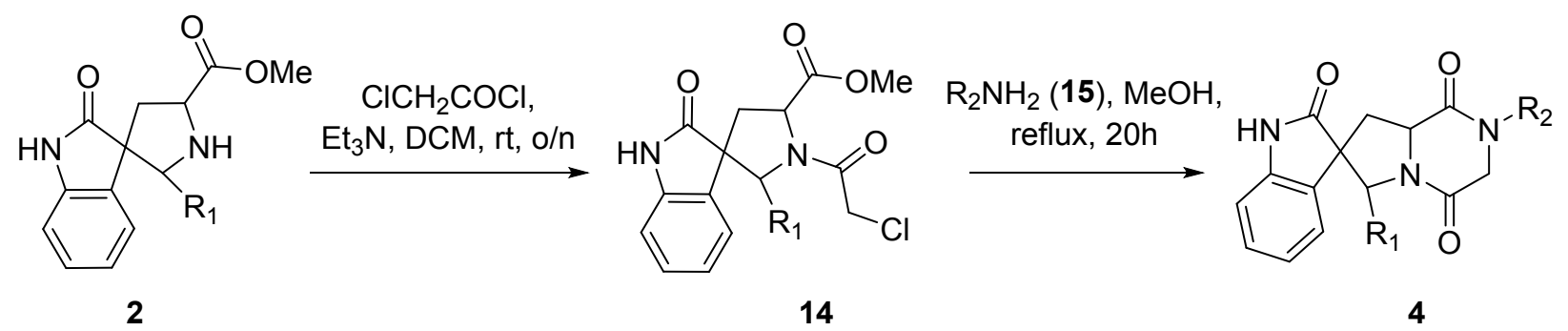

The methyl-2'-alkyl-2-oxospiro[indoline-3,3'-pyrrolidine]-5'-carboxylates 2 were prepared according to literature. ${ }^{5}$ A $50 \mathrm{ml}$ flask was charged with the intermediate $2(5 \mathrm{mmol}, 1$ equiv.) dissolved in dimethylacetamide (stock solutions were prepared and distributed into the 96-position Remp racks). Triethylamine (1.2 equiv.) and chloroacetyl chloride (1.1 equiv.) were added and the reaction mixture was magnetically stirred overnight at room temperature $\left(20-25^{\circ} \mathrm{C}\right)$. $600 \mu \mathrm{L}$ of the reaction mixture of $\mathbf{1 4}(0.1 \mathrm{mmol})$ was transferred into a $0.5-2 \mathrm{~mL}$ microwave vial. Amine $15(0.2$ mmol) and dimethylacetamide $(600 \mu \mathrm{L})$ were added and the reaction mixture was stirred 45 min at $165{ }^{\circ} \mathrm{C}$ under microwave irradiation using our proprietary microwave factory. ${ }^{12}$ The reaction mixture was directly purified by preparative LC/MS.

All the obtained products were characterized by HPLC and MS. Please note that for intellectual property reasons only a part of the structures out of the complete library are listed with their analytical data (the not listed compounds are actively used for screening and their analytical data can therefore not be published).

Product characterization

$\begin{array}{ccccccc}\text { Product } 4 & \begin{array}{c}\text { Collected } \\ {[\mathrm{MW}+\mathrm{H}]^{+}}\end{array} & \mathrm{t}_{\mathrm{R}}(\mathrm{min}) & \begin{array}{c}\text { Yield } \\ (\mathrm{mg})\end{array} & \text { Yield (\%) } & \text { Purity (\%, UV) } & \text { Gradient } \\ \mathbf{4}\{1,5,44\} & 468.62 & 4.27 / 4.50 & 22.3 & 48 & 100 & 1 \\ \mathbf{4}\{1,6,5\} & 470.59 & 5.50 & 8.2 & 17 & 100 & 4 \\ \mathbf{4}\{1,7,41\} & 501.65 & 3.50 & 24.7 & 49 & 100 & 3 \\ \mathbf{4}\{2,1,1\} & 404.49 & 5.92 & 11.8 & 26 & 88 & 2 \\ \mathbf{4}\{2,1,18\} & 422.50 & 4.52 & 22.7 & 54 & 100 & 1 \\ \mathbf{4}\{2,3,44\} & 454.59 & 4.37 & 26.4 & 52 & 90 & 1 \\ \mathbf{4}\{2,4,9\} & 419.50 & 4.68 / 4.99 & 19.4 & 46 & 99 & 1 \\ \mathbf{4}\{2,4,19\} & 408.48 & 5.09 / 5.52 & 6.3 & 15 & 100 & 2 \\ \mathbf{4}\{2,4,41\} & 439.57 & 3.67 / 3.93 & 25.9 & 58 & 99 & 2 \\ \mathbf{4}\{2,5,6\} & 452.96 & 4.92 & 13.3 & 29 & 100 & 4\end{array}$

Note: the spirocyclic intermediates of $\mathbf{4}\{1,4,44\}, \mathbf{4}\{2,4,9\}, \mathbf{4}\{2,4,41\}$ and $\mathbf{4}\{2,4,19\}$ (i.e. $\mathbf{2}\{1\}$ and $\mathbf{2}\{2\}$ ) were diastereomeric mixtures, leading therefore to diastereomeric products. 
NMR and LC-MS spectra for representative compounds

\section{${ }^{1} \mathrm{H}-\mathrm{NMR}\left(400 \mathrm{MHz}, \mathrm{CDCl}_{3}\right)$ and ${ }^{13} \mathrm{C}-\mathrm{NMR}(100 \mathrm{MHz}, \mathrm{MeOD})$ of $3\{1,1,1\}$}
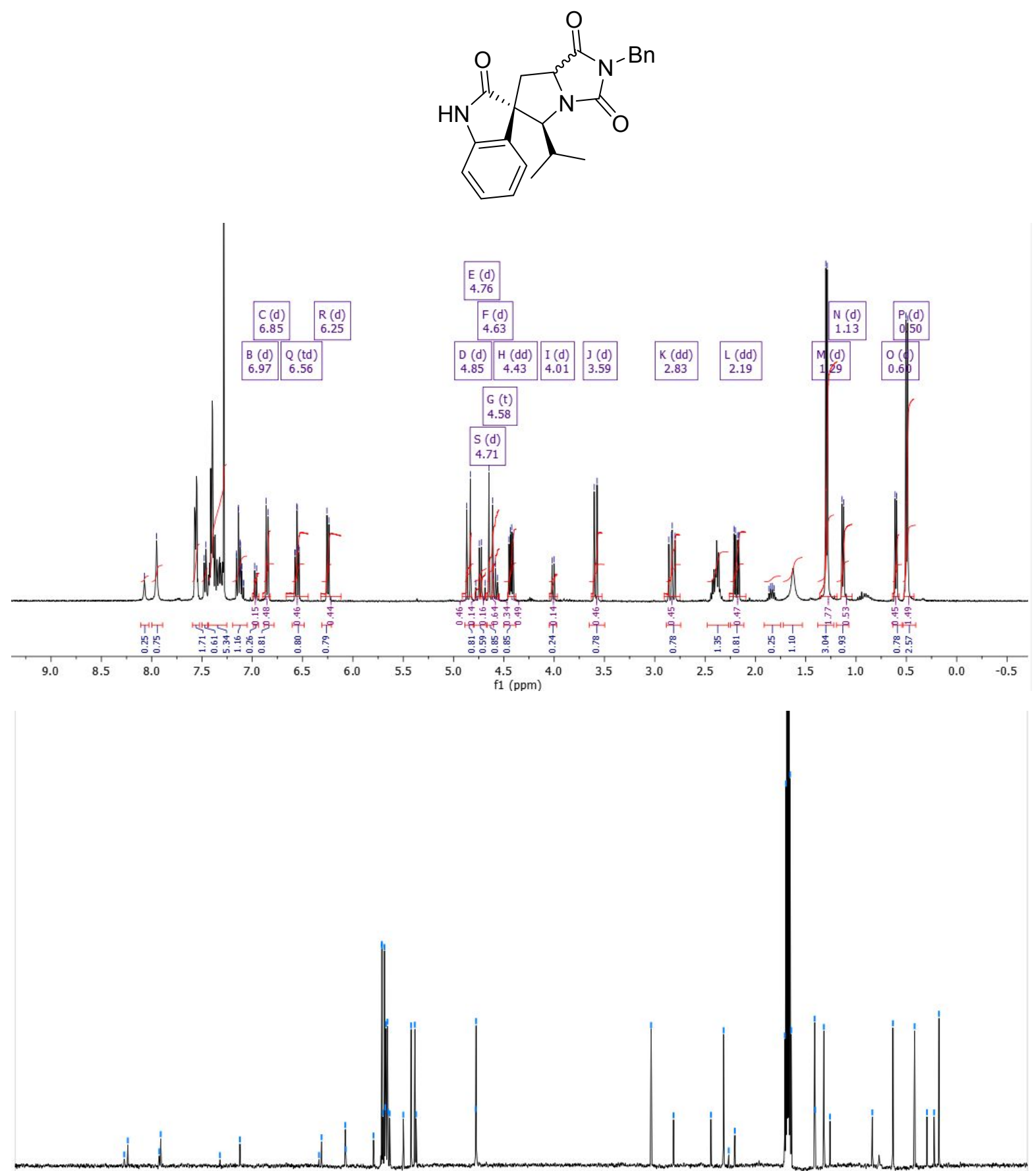

$\begin{array}{llllllllll}190 & 180 & 170 & 160 & 150 & 140 & 130 & 120 & 110 & 100 \\ \mathrm{f} 1(\mathrm{pDm})\end{array}$ 
${ }^{1} \mathrm{H}-\mathrm{NMR}(400 \mathrm{MHz}, \mathrm{MeOD})$ and ${ }^{13} \mathrm{C}-\mathrm{NMR}(100 \mathrm{MHz}, \mathrm{MeOD})$ of $4\{1,1,1\}$

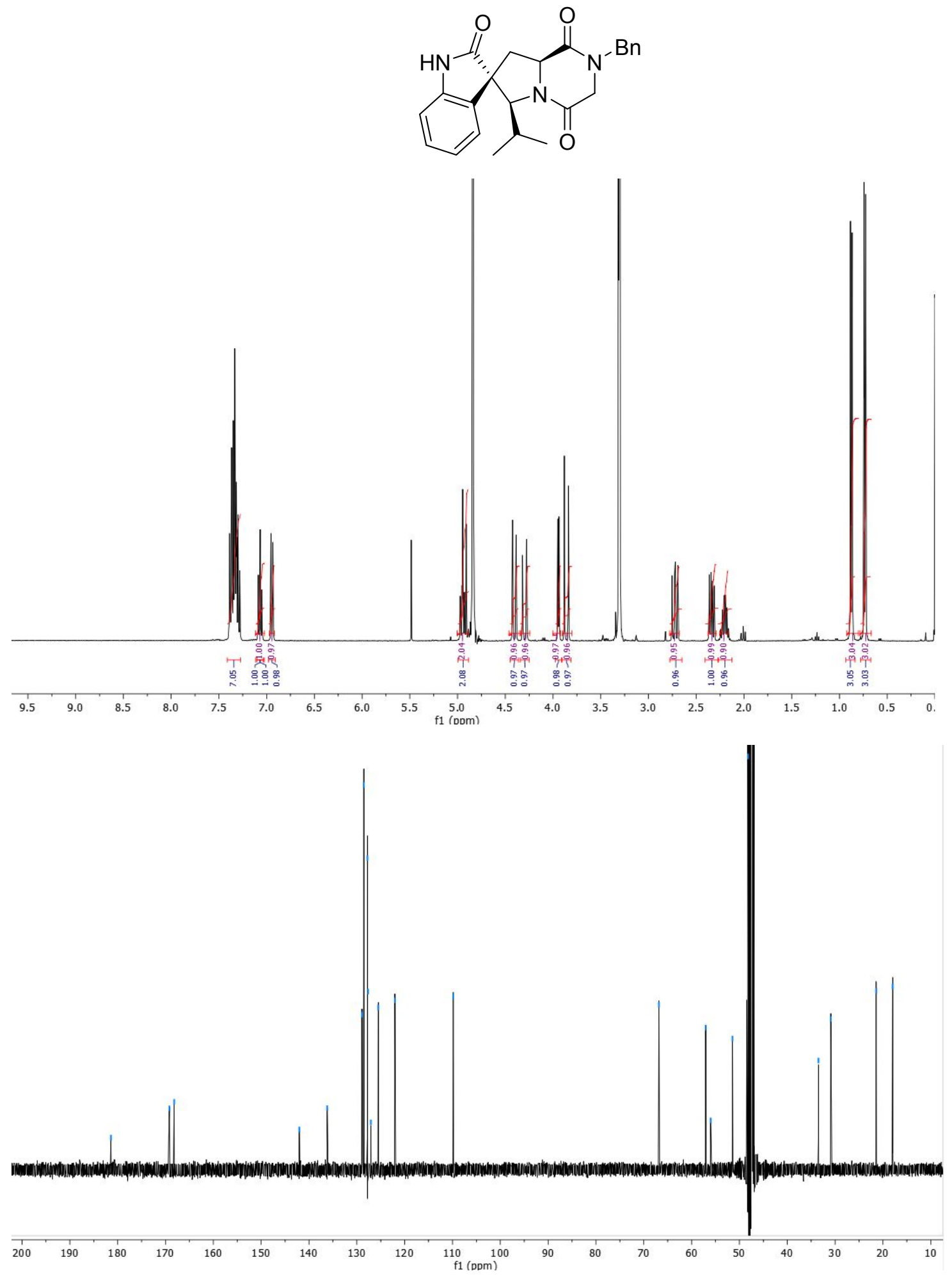


HPLC and MS spectra of selected 5,5-spirocyclic indolinone library members

Note: UV trace of analytical HPLC, MS data from preparative run

$\mathbf{3}\{1,1,15\}$
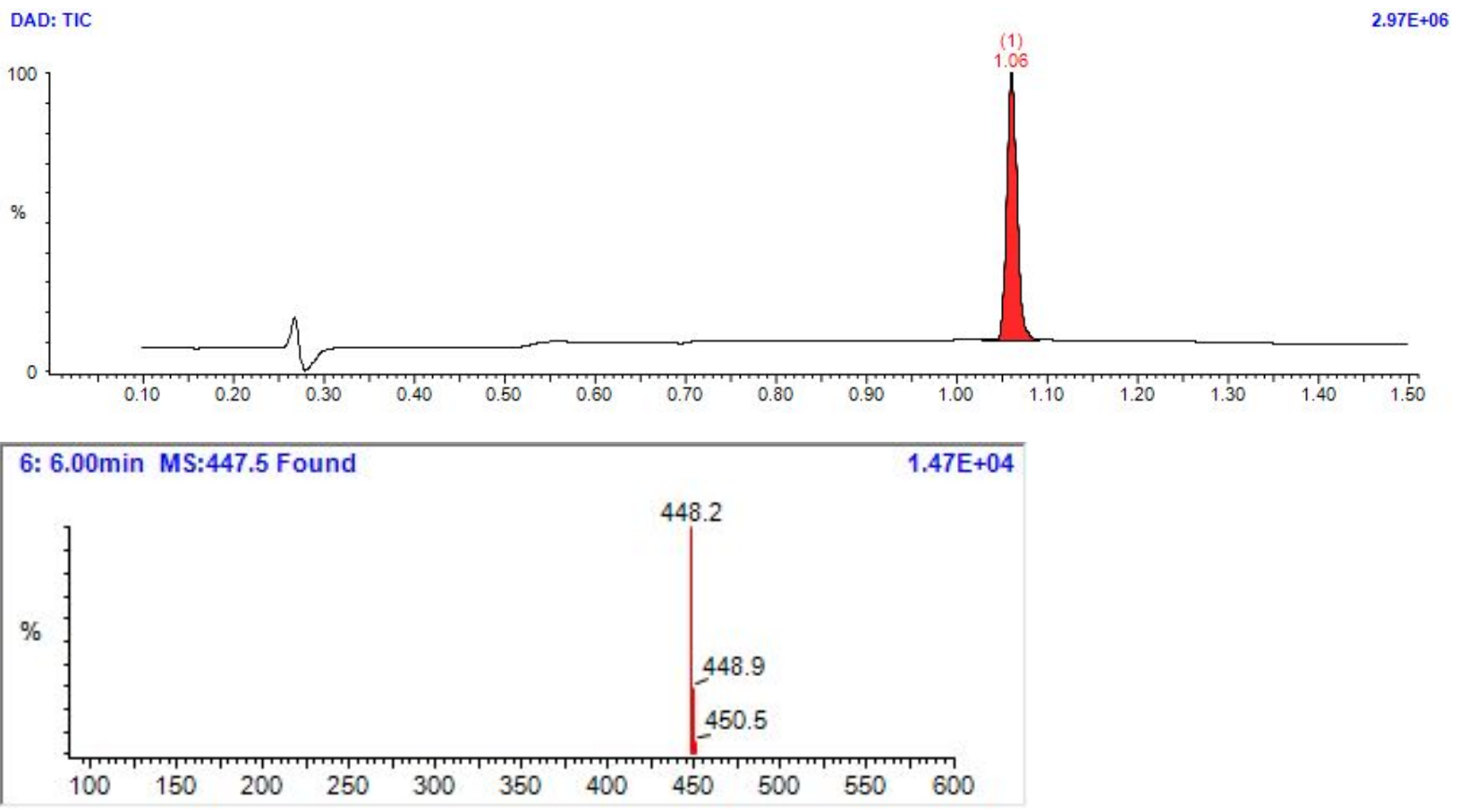

\section{$3\{2,1,21\}$}
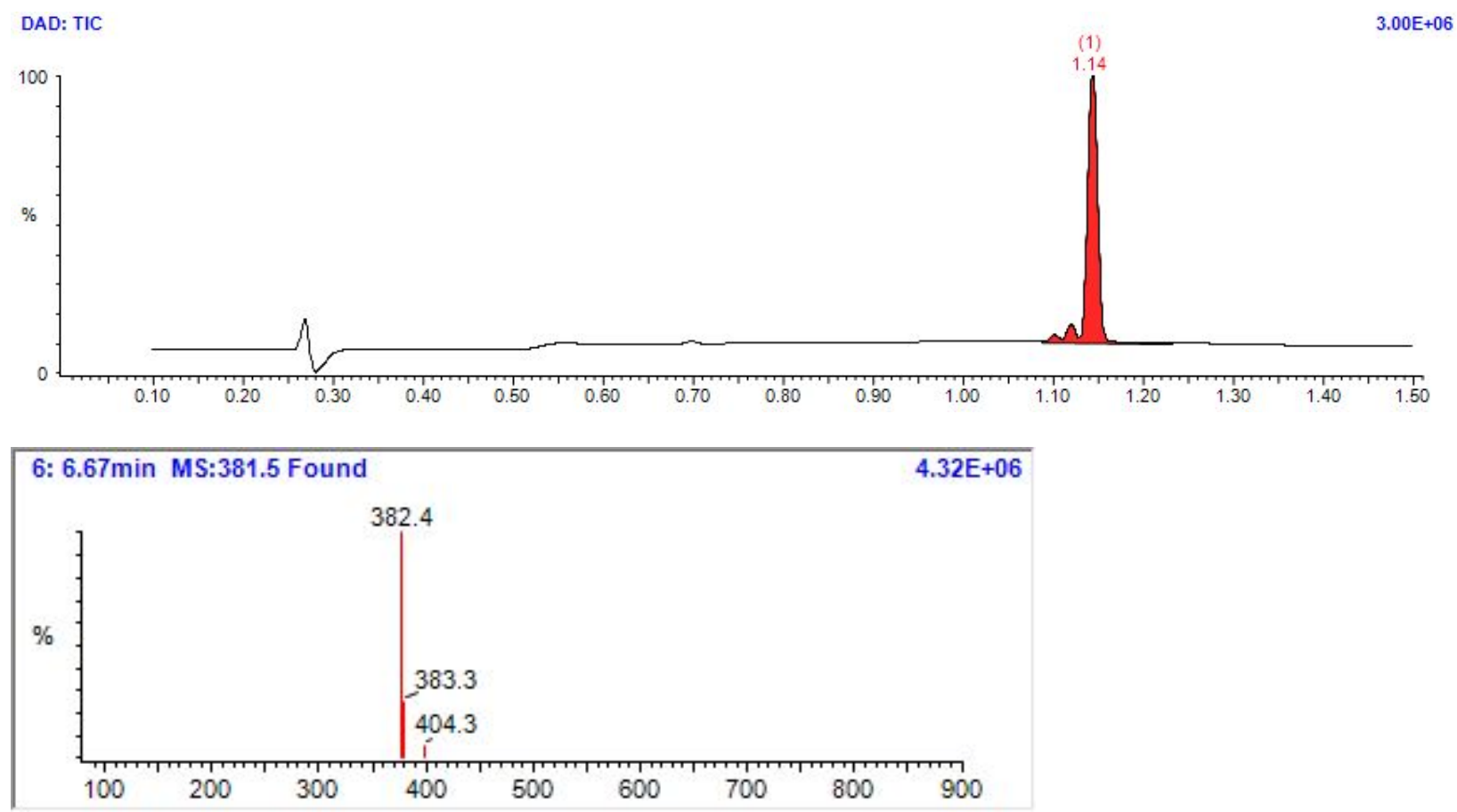
$3\{2,5,14\}$

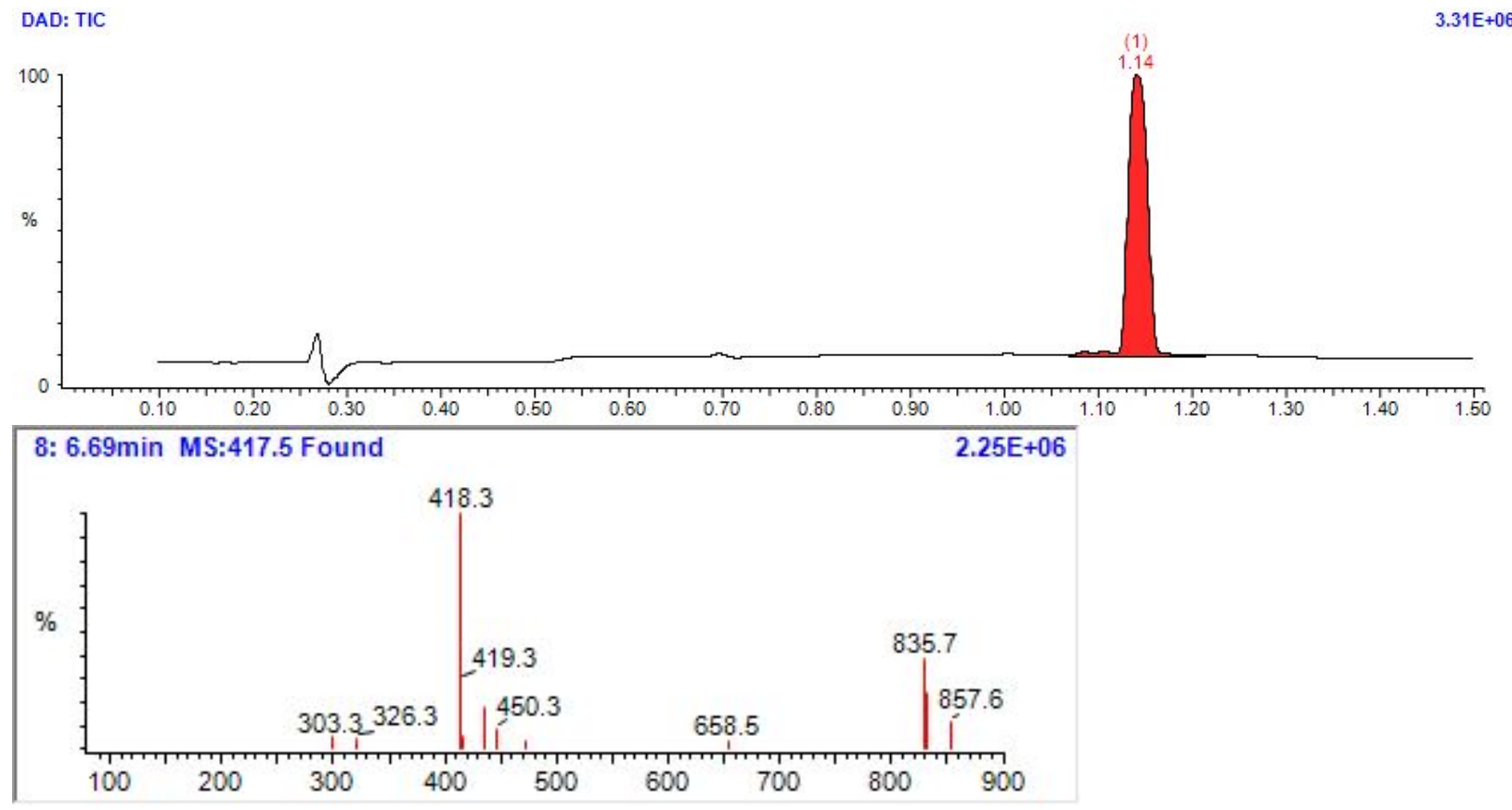

$3\{1,7,17\}$

DAD: TIC
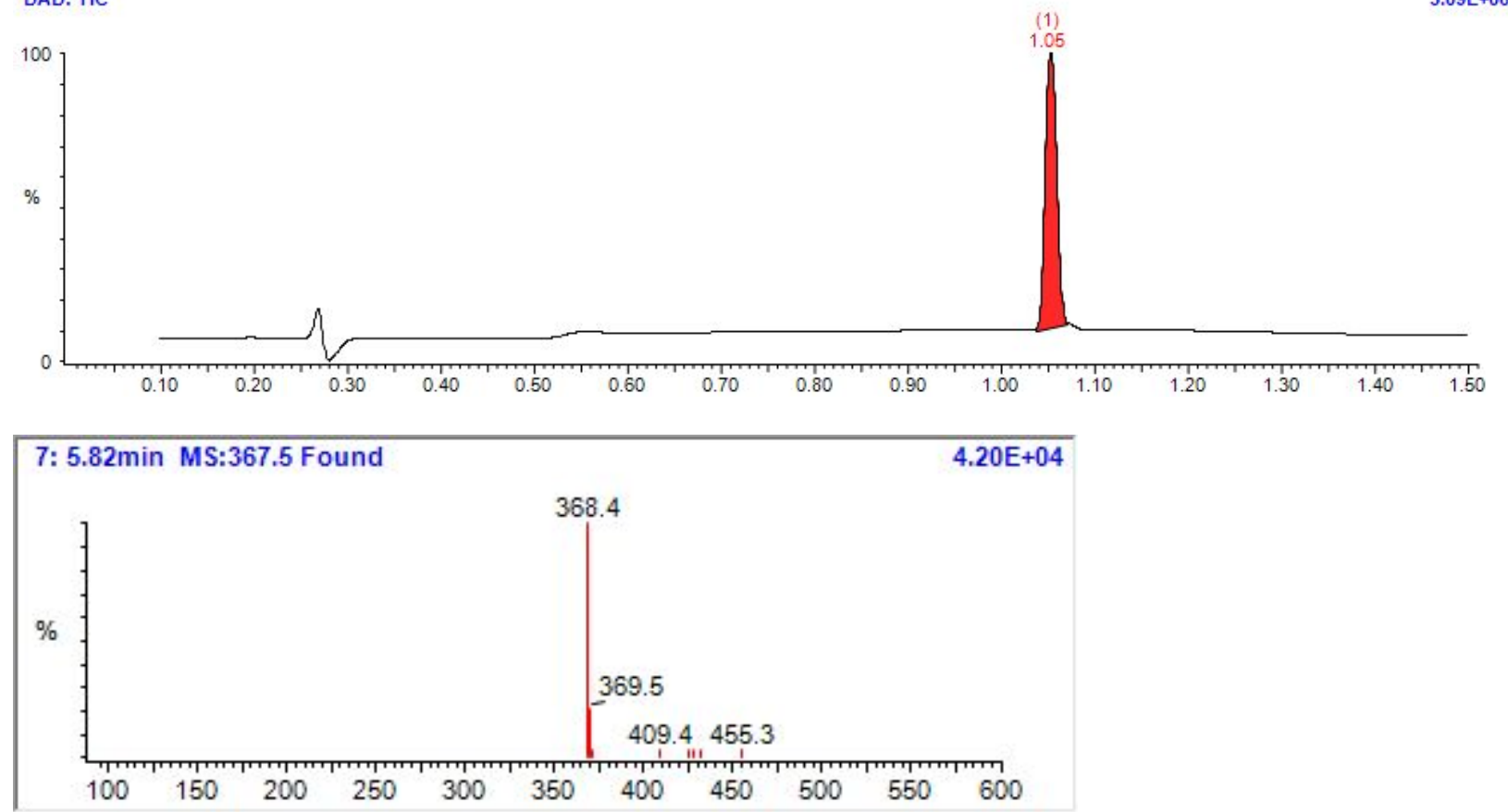
$3\{2,4,16\}$

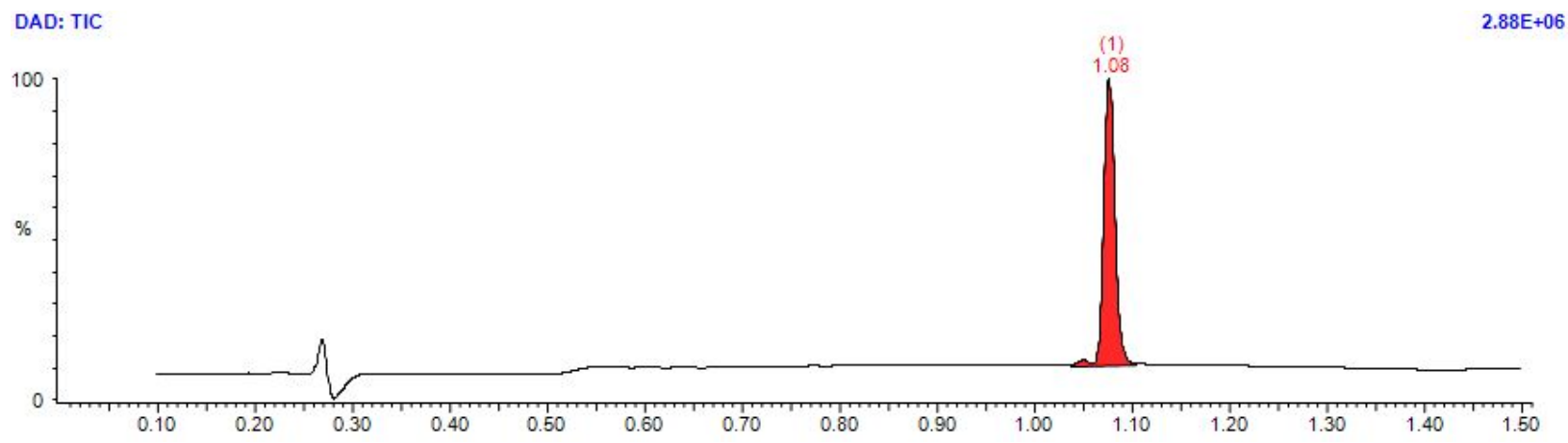

4: 6.09min MS:409.5 Found


HPLC and MS spectra of selected 5,6-spirocyclic indolinone library members

Note: UV trace of analytical HPLC, MS data from preparative run
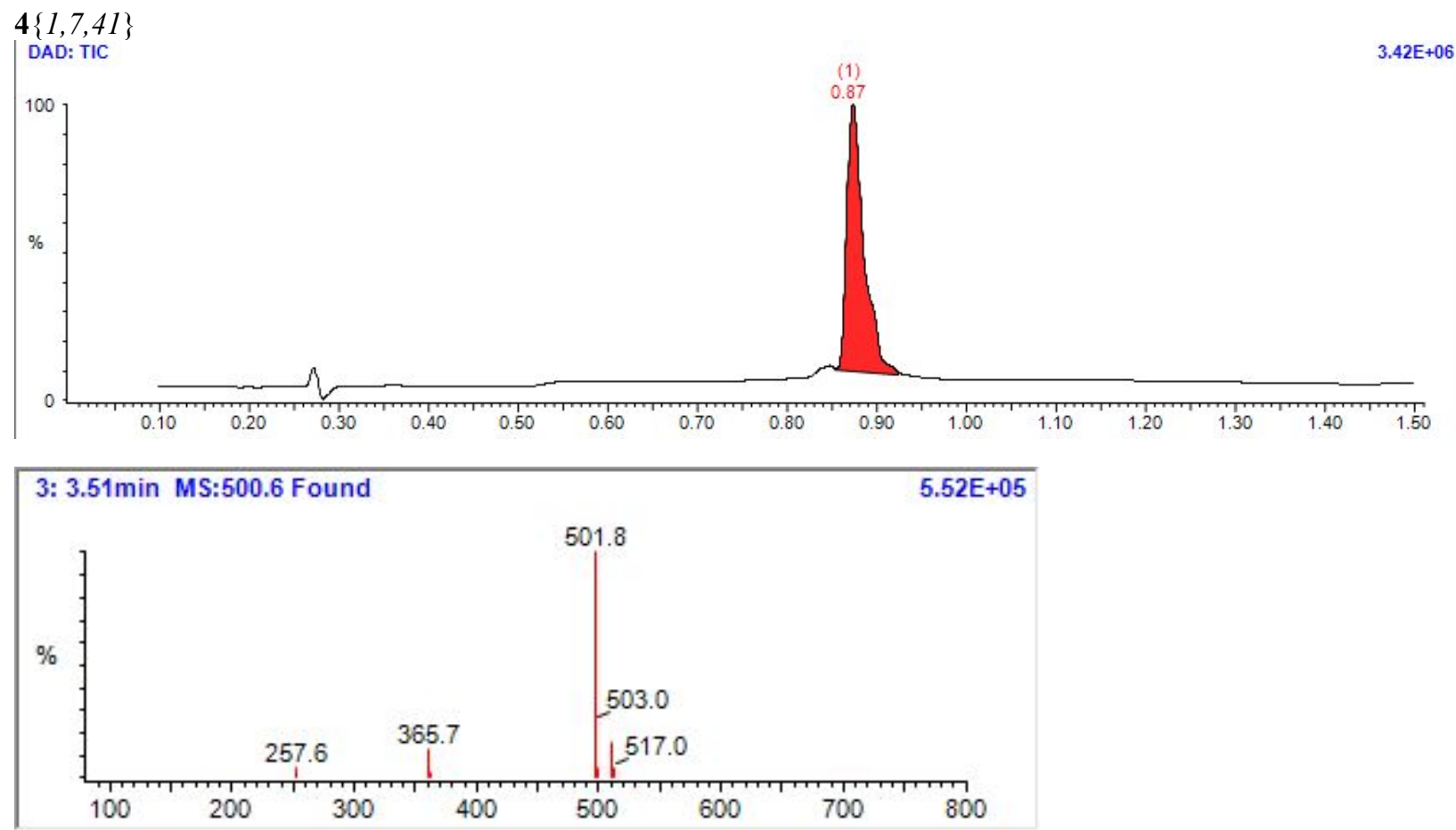

$4\{2,4,9\}$

DAD: TIC
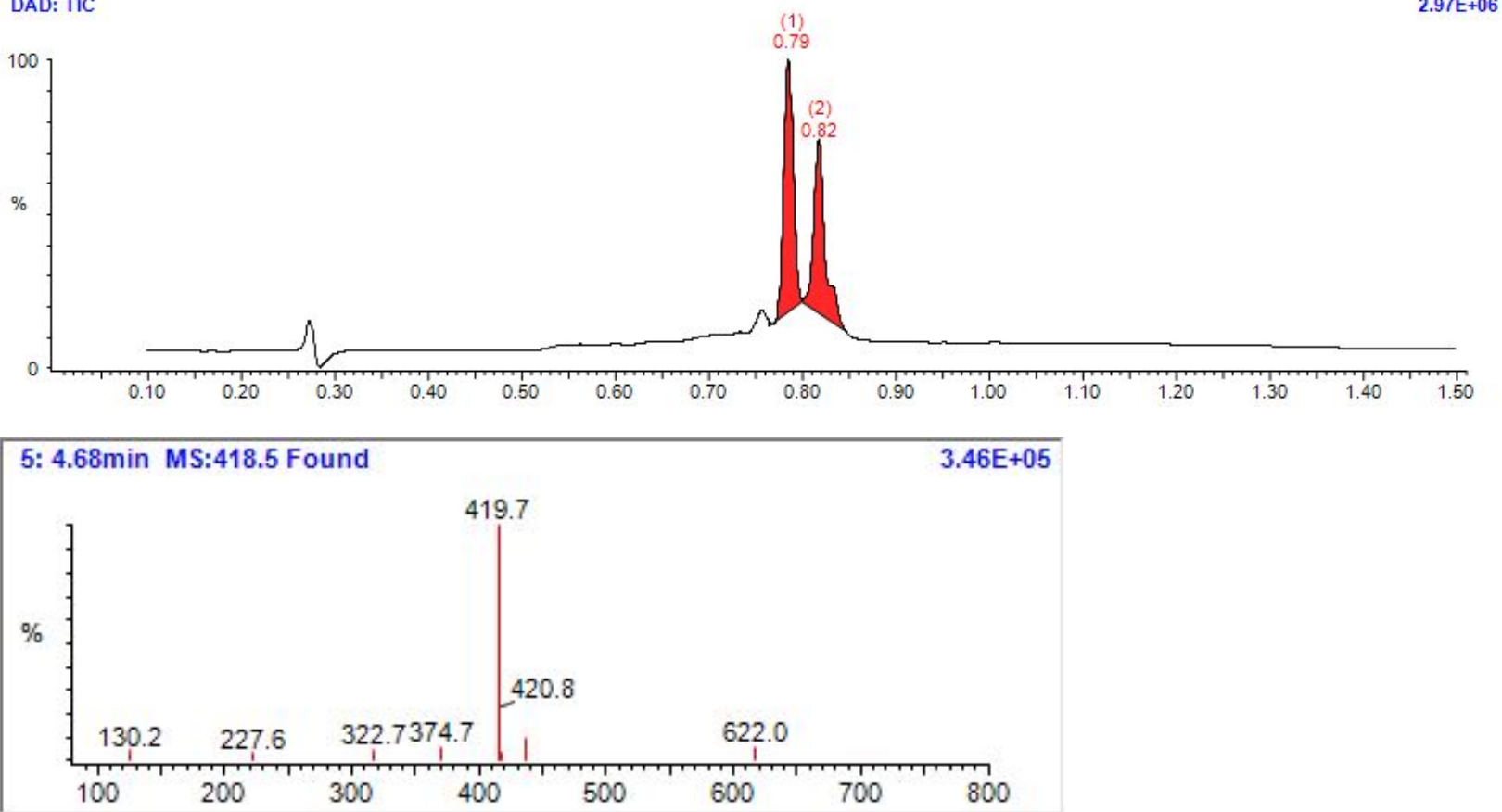


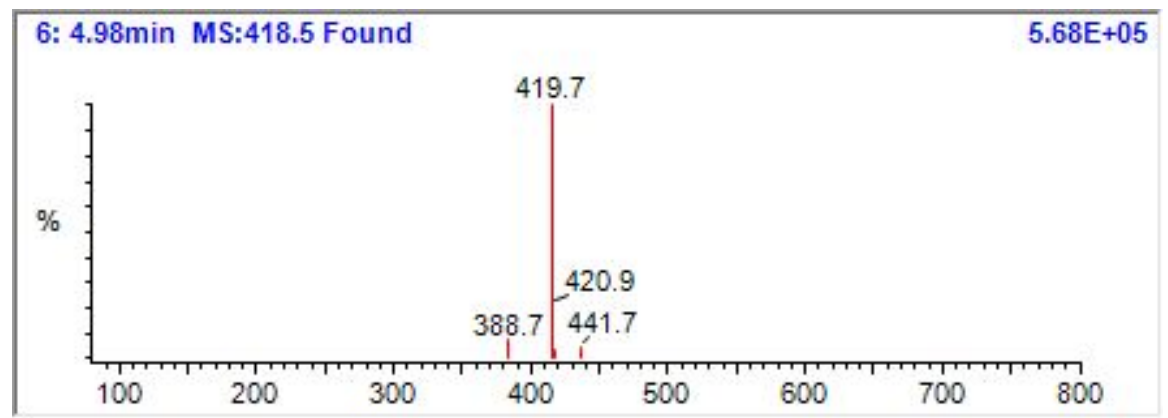

$4\{2,4,41\}$
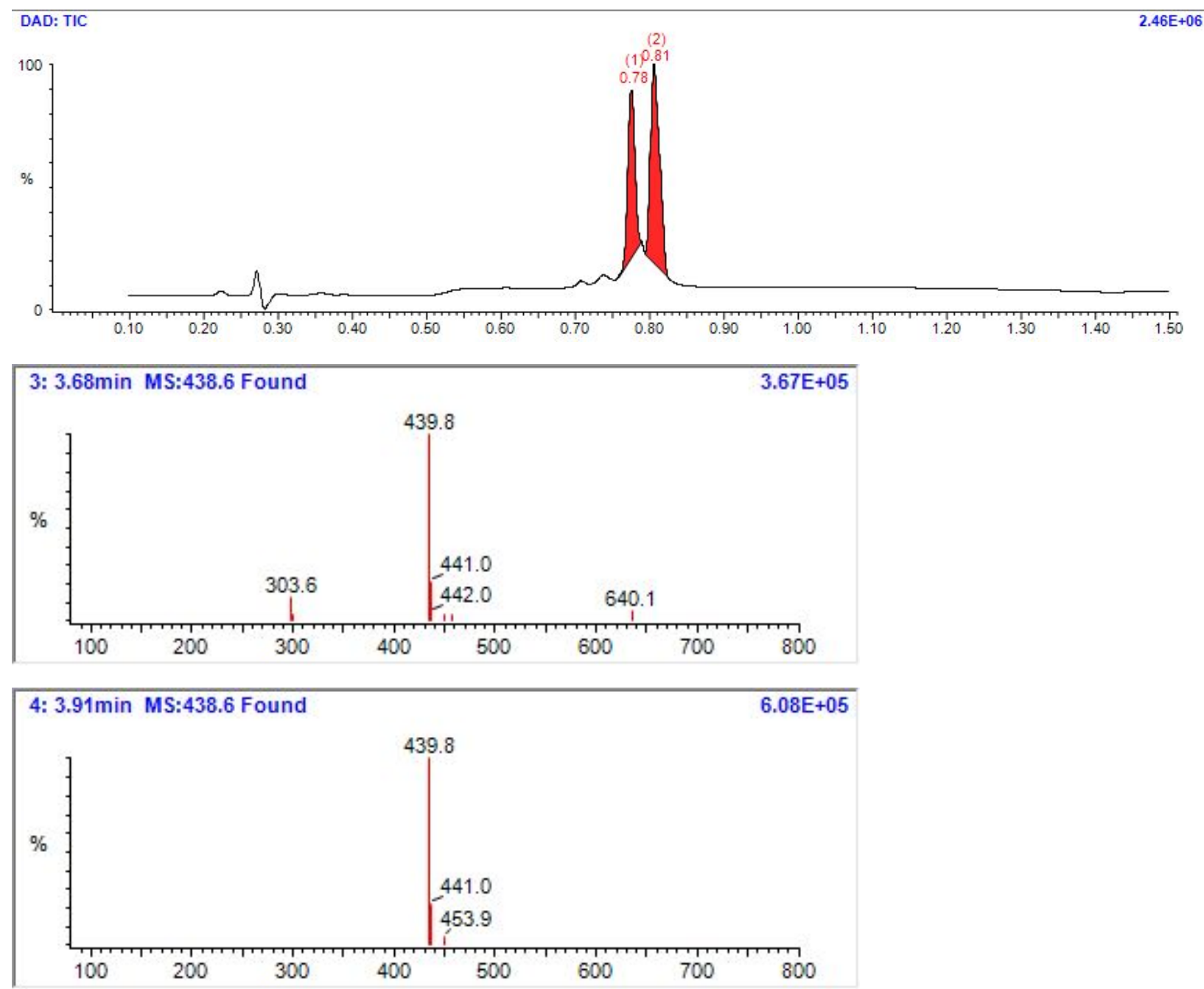
$4\{2,3,44\}$

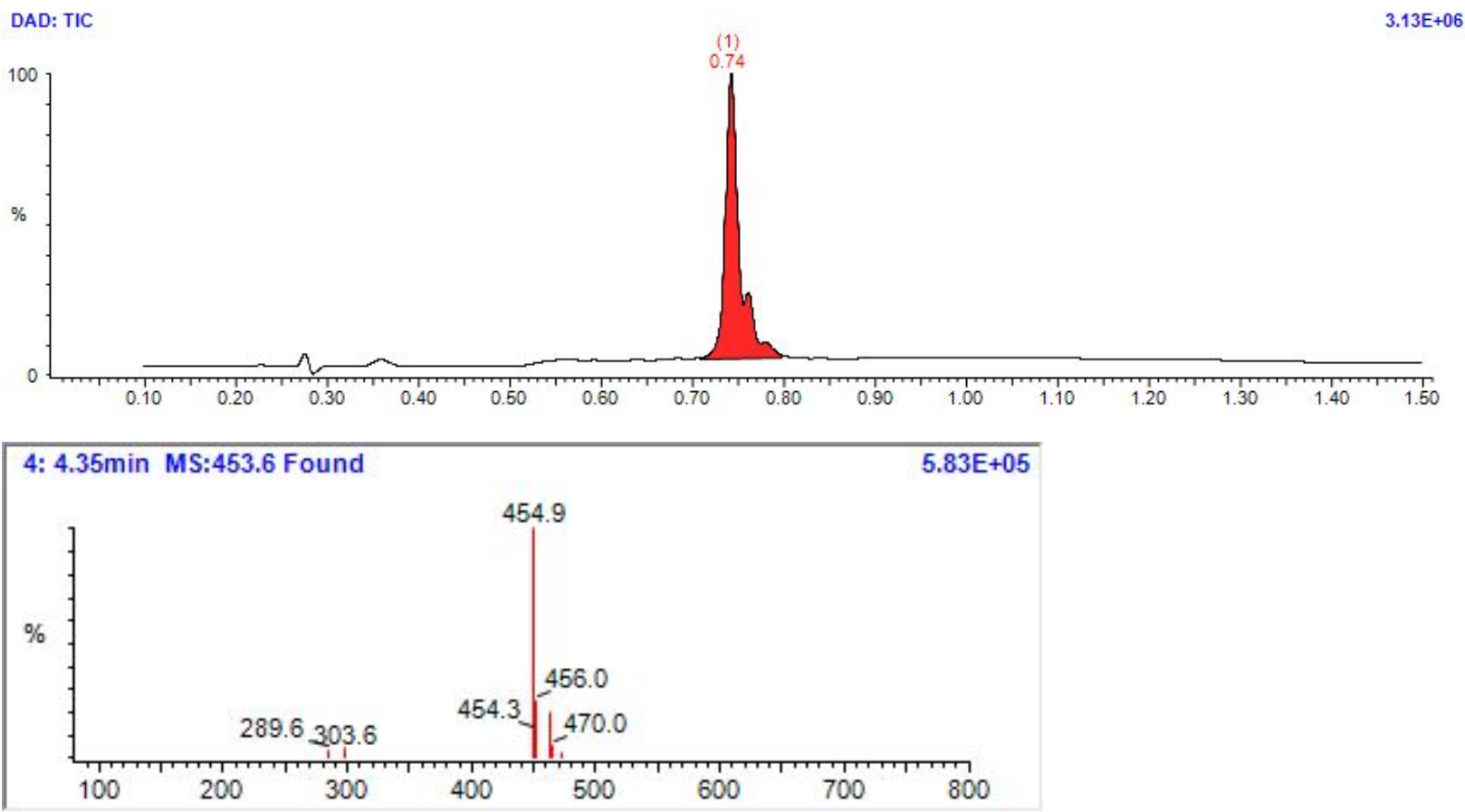

$4\{1,6,5\}$
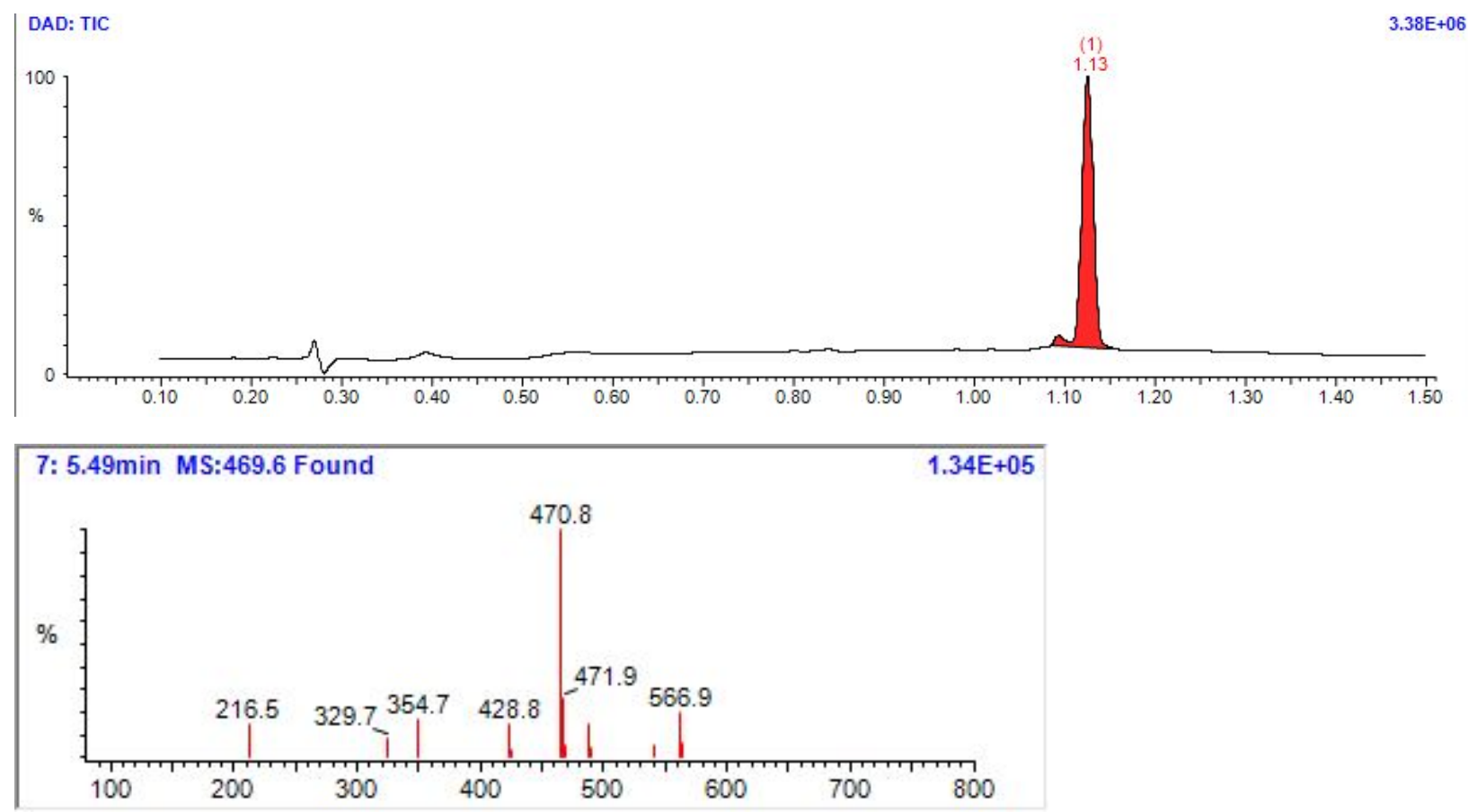


\section{Computational protocol}

We used Maestro 2018-3 (www.schrodinger.com) to perform all the molecular modelling, starting from 3D structures generated by CORINA. Conformational scans were performed using MACROMODEL accessible via the Maestro 2018-3 interface with default settings. We considered a 10 degrees increment for T1 and T2 of both ring systems. Each conformer was submitted to 500 steps of PRCG minimization. Its energy was evaluated using the OPLS2005 force field with the GB/SA solvent model of water. For each prototype compound, representative conformers within $2 \mathrm{kcal} / \mathrm{mol}$ of the minimum were selected for geometry optimization at the density functional theory (DFT) level (B3LYP 6-3-1G*+) using JAGUAR available via the Maestro 2018-3 interface, with default settings. The optimized geometries served as input for single point DFT calculations (B3LYP 6-3-1G*+) in the gas phase and in the Minnesota water solvation model SM8.

Finally, we used the field-based alignment tool Forge 10.5.5 to align the optimized geometries mentioned above with the CSD entry OSULEU.

Maestro: Small-Molecule Drug Discovery Suite 2018-3, Schrödinger, LLC, New York, NY, 2018.

CORINA: Sadowski, J.; Gasteiger, J.; Klebe, G. Comparison of Automatic Three-Dimensional Model Builders Using 639 X-Ray Structures. J. Chem. Inf. Comput. Sci. 1994, 34, 1000-1008.

OPLS 2005: Jorgensen, W.L.; Maxwell, D.S.; Tirado-Rives, J., Development and Testing of the OPLS All-Atom Force Field on Conformational Energetics and Properties of Organic Liquids. J. Am. Chem. Soc. 1996, 118 (45), 11225-11236.

MacroModel: Schrödinger Release 2018-3: MacroModel, Schrödinger, LLC, New York, NY, 2018.

JAGUAR: Bochevarov, A.D.; Harder, E.; Hughes, T.F.; Greenwood, J.R.; Braden, D.A.; Philipp, D.M.; Rinaldo, D.; Halls, M.D.; Zhang, J.; Friesner, R.A., Jaguar: A high-performance quantum chemistry software program with strengths in life and materials sciences. Int. J. Quantum Chem. 2013, 113 (18), 2110-2142.

Forge: Forge, 10.5.5, Cresset $\AA$, Litlington, Cambridgeshire, UK. 